\title{
Cochlear and Vestibular Functional Study in Patients with Sudden Deafness and Lyme Disease
}

\author{
Ziane Selmani ${ }^{1}$, Ilmari Pyykkö ${ }^{2}$ \\ ${ }^{1}$ Health Centre of Aava, Kerava, Finland \\ ${ }^{2}$ Department of Otolaryngology, University Hospital of Tampere, \\ University of Tampere, Tampere, Finland \\ Email: ziane_selmani@hotmail.com
}

Received November 7, 2013; revised December 5, 2013; accepted January 2, 2014

Copyright (C) 2014 Ziane Selmani, Ilmari Pyykkö. This is an open access article distributed under the Creative Commons Attribution License, which permits unrestricted use, distribution, and reproduction in any medium, provided the original work is properly cited. In accordance of the Creative Commons Attribution License all Copyrights (C) 2014 are reserved for SCIRP and the owner of the intellectual property Ziane Selmani, Ilmari Pyykkö. All Copyright (C 2014 are guarded by law and by SCIRP as a guardian.

\begin{abstract}
Lyme disease (LD) and idiopathic sudden deafness (ISD) are supposed to be different diseases with different aetiologies. In an attempt to confirm this assumption, 10 patients with confirmed LD and 12 patients with ISD were consecutively included into the study. Further to the laboratory and audio logical investigation, a low frequency sound (LFS) stimulation on posturography was performed and evaluated. Patients with ISD had higher pure tone average (PTA) than patients with LD (PTA ISD/LD was 72 decibel (dB)/23 dB). There was no difference of vertigo between LD and ISD patients. Patients with ISD have more body sway velocity during the first stimulation than patients with LD. We conclude that the cochlear organ is more affected in ISD patients than in patients with LD. However, the vestibular organ seems to be affected in both diseases. ISD and LD are two different disease entities with different aetiologies but with common otological clinical signs.
\end{abstract}

\section{KEYWORDS}

Idiopathic Sudden Deafness; Lyme Disease; Low Frequency Sound Stimulation

\section{Introduction}

Lyme disease (LD) and idiopathic sudden deafness (ISD) are supposed to be two different disease entities with different ethiopathogenic factors. It is known that the LD has the ability to affect the ear balance and the hearing organs of the inner together or separately [1]. ISD involves the hearing organ principally but can also involve the inner ear balance organ during certain conditions. Literature suggests that sudden sensorineural hearing loss may coincide with Borrelia burgdorferi infection [2]. A higher incidence of confirmed positive serology for Borrelia burgdorferi in patients with sudden deafness seems to be depending on the country and on the tests used to confirm Lyme disease [3]. On the basis of clinical standpoints, clinical inner ear manifestations are common in both diseases $[4,5]$. Both diseases have shared symptoms like sensorineural hearing loss, tinnitus and vertigo which on the other hand can be also encountered in different inner ear disorders. Inner ear manifestations during LD are a result of an inflammation triggered by an immunological reaction occurring in the cochlea following a tick-bite (by Ixodes racinus) [5]. Sudden deafness is a sharing symptom of various inner ear disorders. It is encountered in Ménière's disease [6], acoustic neurinoma [7], perilymphatic fistula [8], and certain autoimmune diseases [9] after viral infection [10]. Furthermore, sudden deafness has been described in LD [3]. It is difficult to estimate the right diagnosis only on the basis of sudden deafness. Hence, there is a need of further evidences based on clinical and ethiopathological differences and laboratory examinations before understanding the two disease entities. Once the right diagnosis is established, it will be more feasible to assess an adequate therapy, to provide possibly a rapid recovery and to avoid the handicap of dizziness and of hearing loss, furthermore, to avoid other morbidities related to LD like myocarditis, keratitis and encephalitis.

The purpose of this study is to find whether there is a 
difference on physiological standpoints by measuring and analysing the hearing level, by performing a posturography to explore the vestibular functional system.

\section{Materials and Methods}

\subsection{Patients}

Ten patients with confirmed Lyme disease (mean age $=$ 42 yrs) were consecutively included into the study. Two patients were excluded from the study because of the worsening of their symptom; hence, they could not participate to this study. Twelve patients (mean age $=58 \mathrm{yrs}$ ) with idiopathic sudden hearing loss (5 right sided, 6 left sided and one with bilateral disease) were admitted to the study. ISD was defined as a hearing loss detected on awakening or other rapid deterioration in hearing acuity occurring in less than two days without barotraumas, head injury or any other aetiologies.

The final diagnosis of the Lyme disease was established on the basis of clinical history, neurological, ENT and ophthalmological examinations and also on the basis of otoneurological and laboratory tests.

Careful investigation into clinical history involved enquiry about the above-mentioned complaints, plus examination including full ENT examination, neurological examination, and inspection of the lymph nodes and skin. Infection with Borrelia Burgdorferi (BB) was suspected when the history included a stay in an endemic area, insect bite, cutaneous lesion, arthralgia or myalgia. Infection was also suspected when examination revealed lymph node enlargement, erythema migrans or cranial nerve involvement (especially the facial and vestibulocochlear nerves). Ophthalmologic consultation was carried out when keratitis was suspected, and it was also taken as a suspicion of infection. All patients underwent: 1) conventional audiometry, 2) brain stem response measurement (BSER), 3) screening for serum antibodies (IgG and IgM) against BB, 4) screening by PCR for borrelial DNA, 5) screening by immunoblot with enzyme-linked immunosorbent assay (ELISA) for immunoglobulin classes and subclasses against various antigens of $\mathrm{BB}$.

Criteria for diagnosis of Lyme disease included erythema migrans, positive PCR or positive serum immunoblot results, in addition to seropositivity. Seropositivity alone was considered insufficient for the diagnosis of Lyme disease and it should be supported by clinical and further laboratory findings, such as immunoblot or PCR results [11].

In attempt to evaluate wether the hearing organ is affected or not in LD and in ISD as well, conventional audiological tests was performed in all patients of the two groups of diseases, also radiological investigation or magnetic resonance imaging of the inner ear were per- formed if necessary.

Air and bone conducted thresholds were examined at frequencies of 0.25 to $8 \mathrm{kHz}$ with Madsen OB-822 diagnostic audiometers and TDH-39 headphones. The audiometers were calibrated according to ISO standards. The pure-tone average (PTA) was used as a variable for hearing loss $(0.5,1,2$ and $4 \mathrm{kHz})$.

As the balance organ is a part of the inner ear system, it can be affected within or without the hearing organ by inner ear diseases. Affection of the inner ear balance organ causes problems and difficulties to the patient to keep normal postural stability at rest or under stimulation conditions.

In attempt to evaluate postural stability of the patients of the two disease groups, low frequency sound stimulation on posturography was performed.

Recording techniques in detail are published elsewhere [12].

For posturography a force platform was used, the patient standing without shoes, heels together, feet in a 30 degree angle and with arms crossed over the chest [13]. The force platform is based on the strain gauge principle [14]. Vertical forces over the platform are measured in reference to fore and aft, and lateral directions, and lateral directions. The patient is instructed not to bend his knees to avoid the generally protective stance. After the initial 20-second sway stabilization, the quiescent stance is recorded during a 30-second period. Measurements are made under visual control (eyes open) and under nonvisual control (eyes closed). A microcomputer (Hewlett Packard $75 \mathrm{C}$ ) controls the stimulator unit, and the vibration is delivered with rotating shakers, giving at all frequencies, $0.4 \mathrm{~mm}$ peak-to-peak amplitude (when hanging free).

Low frequency sound (LFS) stimulation:

The investigation started with evaluation of the vestibulospinal responses when loading the ear with LFS stimulation. Both ears were studied separately. The LFS stimulation was generated with a piston type air sampling pump (MSA, USA), which has an adjustable stroke frequency ranging from 6 to $80 \mathrm{~Hz}$. The frequencies used in the test were 25, 50 and $63 \mathrm{~Hz}$ at a sound pressure level of $130 \mathrm{~dB}$ for $25 \mathrm{~Hz}, 132 \mathrm{~dB}$ for 50 and $63 \mathrm{~Hz}$ respectively.

The posturography lasts 180 seconds. To fore and aft, and lateral body sway signals are processed in a microcomputer (Hewlett Packard 9000 series 300). The data were sampled at $33.3 \mathrm{~Hz}$ frequency, and to eliminate artefacts, filtered with a non-linear 3 point median filter, smoothened with linearly moving average filters of 3 and 5 points to remove random noise [14]. The program calculates sway velocity (SV).

The study was approved by the ethical committee of the University of Helsinki (04/94). 


\subsection{Statistical Analysis}

Differences between the two groups of diseases on the basis of the level of hearing loss, the results of body sway on posturography was analysed by using the student T-test. The analysis of difference between two groups in terms of the presence of vertigo was reanalyzed by Fisher-test. Findings were considered to be statistically significant when $\mathrm{p}$ was less than 0.05 .

\section{Results}

Patients with ISD had higher pure tone averages than the patients with Lyme disease. The mean average of PTA in patients with LD was $23 \mathrm{~dB}$ and in patients with ISD, it was $72 \mathrm{~dB}(\mathrm{p}<0.05)$, (Figure 1 ).

There was no difference in the appearance of vertigo between the patients with LD and those with ISD (Table 1), $(\mathrm{p}>0.05)$.

There were no differences between body sway velocities at rest between the patients with Lyme disease and those with ISD (Figure 2), ( $>0.05)$.

Patients with ISD have higher body sway velocities at the first LFS stimulation than the patients with LD (Figure 3), $(\mathrm{p}<0.05)$.

\section{Discussion}

Otolaryngological manifestations are common in Lyme disease, affecting up to $75 \%$ of the patients [3]. In our study, the hearing loss was more profound in the patients with ISD than in the patients with LD. The cochlea seems to be a site of derangement in case of ISD than in case of LD. This is probably due to the predominance selective ethiopathogeneicity of causative agent regarding the cochlea in case of ISD. It has been reported also that LD can provoke progressive and sudden hearing loss.

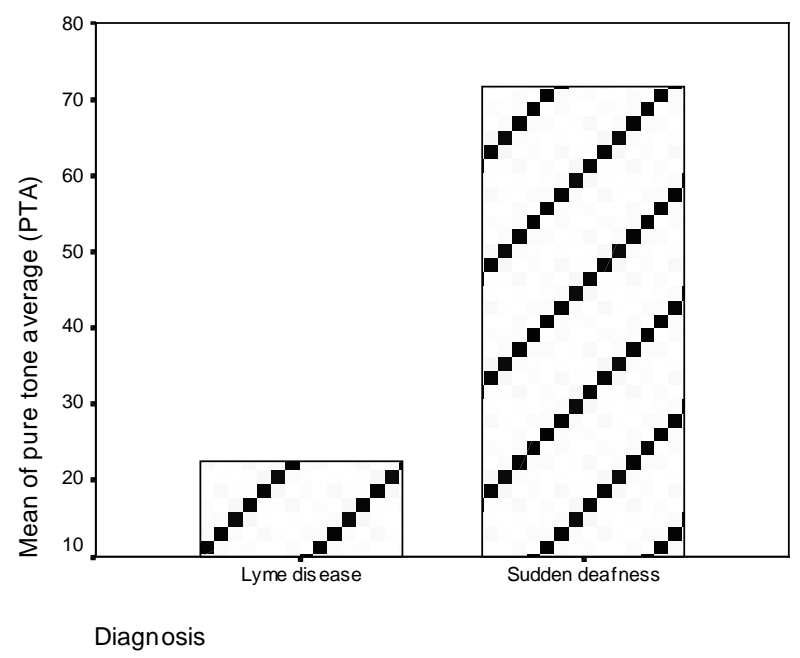

Figure 1 . The pure tone average $(0.5,1,2,4 \mathrm{kHz})$ in patients with Lyme disease and sudden deafness.
Table 1. The percentage of patients with and without vertigo in Lyme disease and sudden deafness groups.

\begin{tabular}{ccc}
\hline Diagnosis & Lyme disease & Idiopathic sudden deafness \\
\hline Number of patients & $8(100 \%)$ & $12(100 \%)$ \\
Right side & $5(62,5 \%)$ & $7(58.3 \%)$ \\
Left side & $3(37,5 \%)$ & $5(41.7)$ \\
Vertigo & $5(62.5 \%)$ & $5(41.7 \%)$ \\
Without vertigo & $3(37.5 \%)$ & $7(58.3 \%)$ \\
\hline
\end{tabular}

$\mathrm{p}=0240057$.

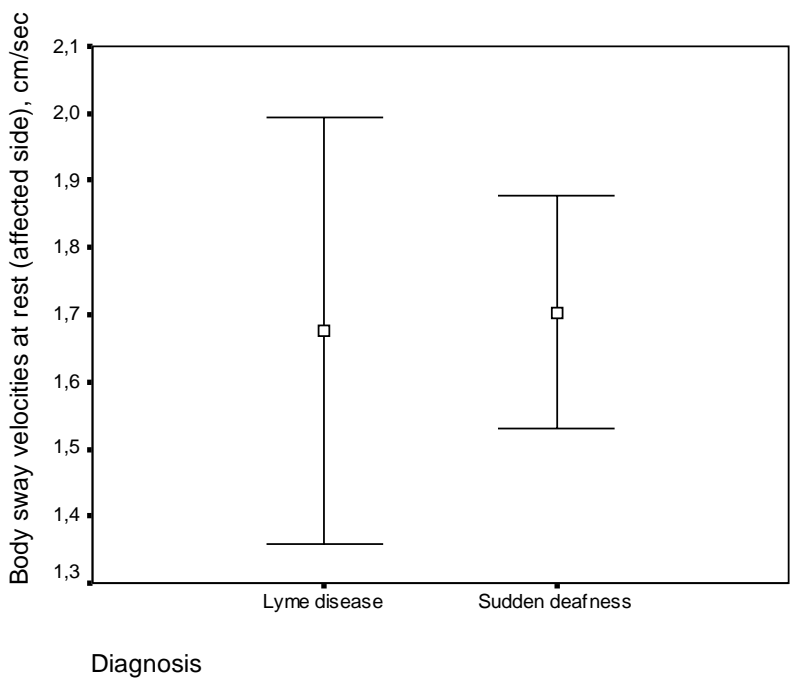

Figure 2. Body sway velocity of patients with Lyme disease and sudden deafness at rest (without stimulation). Mean and standard deviation of body sway velocity are given.

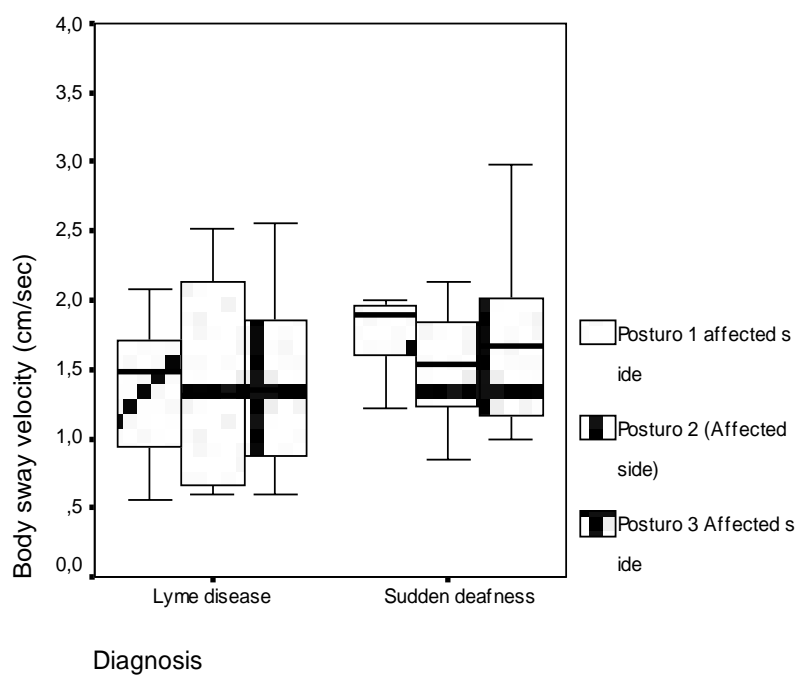

Figure 3. Body sway velocity of Lyme disease and sudden deafness patients during low frequency sound stimulation of the affected side. Mean and standard deviation of body sway velocity are given. 
Hearing loss in LD could be a result of inflammation triggered by an immunological reaction occurring in the cochlea following BB infection [5]. The immunological reaction is manifested first by the elevation of IgM levels after 2 weeks of infection, with a peak at $4-6$ weeks before it starts to fall and will disappear two weeks later [15]. The levels of IgG start to rise at 6 weeks and they stay high for months to years. It is known that Lyme disease is a systemic disease which can cause sensorineural hearing loss $[5,16]$ which can be related to the immunological reaction causing then cochlear vasculitis.

Vertigo was almost equally present in both groups of diseases. It was present in 38\% of patients with LD and in $41 \%$ patients with ISD. Ishizaki et al. (1991) have reported that vertigo was the predominant symptom in patients with confirmed LD. In the majority of the cases, the disease resembles vestibular neuronitis in the acute stage [17]. Other studies have reported that the incidence of seropositivity against B. Burgdorferi among the vertigo patients did not differ from the incidence of the normal population [11].

In our study, the patients with ISD have pathological body sway reactions to the first LFS stimulation on posturography. However, there was no difference on postural stability between the patients with LD and ISD during the second and the third stimulation. This could be a sign of a slight derangement of the vestibulo-spinal reflex, which has been more apparent during the first LFS stimulation before it started to be stabilised during the second and third LFS stimulations. Hence, during ISD and furthermore to the derangement of cochlear system, the vestibular system can also be affected. By the fact that patient with ISD has presented a pathological body sway only during the first LFS stimulation and not during the second and to the third stimulation. This might be due to an eventual correction or compensation of the vestibular organ which has occurred during the second and third LFS stimulations. This is a sign that the vestibular system is still working and maintaining its functional integrity and its adaptive property.

\section{Conclusion}

In conclusion, the cochlear organ is more affected in ISD than in LD. However, the vestibular organ seems to be affected in both diseases. ISD and LD are two different disease entities with different aetiologies but with common otological signs by the fact that both diseases have a common target which is the inner ear.

\section{REFERENCES}

[1] L. E. Walther, H. Hentschel, A. Oehme, H. Gudziol and E. Beleites, "Lyme Disease-A Reason for Sudden Sensorineural Hearing Loss and Vestibular Neuronitis?”
Laryngo-Rhino-Otologie, Vol. 82, No. 4, 2003, pp. 249257. http://dx.doi.org/10.1055/s-2003-38938

[2] N. Peeters, B. Y. van der Kolk, S. F. Thijsen and D. R. Colnot, "Lyme Disease Associated with Sudden Sensorineural Hearing Loss: Case Report and Literature Review,” Otology \& Neurotology, Vol. 34, No. 5, 2013, pp. 832-837. http://dx.doi.org/10.1097/MAO.0b013e31827c9f93

[3] M. C. Lorenzi, R. S. Bittar, M. E. Pedalini, F. Zerati, N. H. Yoshinari and R. F. Bento, "Sudden Deafness and Lyme Disease,” Laryngoscope, Vol. 113, No. 2, 2003, pp. 312-315. http://dx.doi.org/10.1097/00005537-200302000-00021

[4] Z. Selmani, I. Pyykkö, H. Ishizaki and N. Ashammakhi, "Use of Electrocochleography in Assessing Endolymphatic Hydrops in Patients with Lyme Disease and Ménière's Disease,” Acta Otolaryngologica (Stockh), Vol. 122, No. 2, 2002, pp. 173-178.

[5] A. L. Moscatello, D. L. Worden, R. B. Nadelman, G. Wormser and F. Lucente, "Otolaryngologic Aspects of Lyme Disease,” Laryngoscope, Vol. 101, No. 6, 1991, pp. 592-595.

http://dx.doi.org/10.1288/00005537-199106000-00004

[6] R. Filipo, A. Cordier, M. Barbara and G. A. Bertoli, "Electrocochleographic Findings: Menière's Disease versus Sudden Sensorineural Hearing Loss,” Acta Otolaryngologica (Stockh), Vol. 117, No. s526, 1997, pp. 21-23. http://dx.doi.org/10.3109/00016489709124015

[7] N. Yanagihara, S. Murakami, M. Asai, T. Hatakeyama and K. Zenke, "Hearing Improvement Following Removal of Acoustic Neuroma with Preoperative Sudden Deafness,” Ear Nose Throat Journal, Vol. 73, No. 12, 1994, pp. 890-894.

[8] V. Goodhill, "Sudden Deafness and Round Window Rupture,” Laryngoscope, Vol. 81, 1971, pp. 1462-1474. http://dx.doi.org/10.1288/00005537-197109000-00010

[9] D. Yehudai, Y. Shoenfeld and E. Toubi, "The Autoimmune Characteristics of Progressive or Sudden Sensorineural Hearing Loss,” Autoimmunity, Vol. 39, No. 2, 2006, pp. 153-158. http://dx.doi.org/10.1080/08916930500499599

[10] R. Mentel, H. Kaftan, U. Wegner, A. Reismann and L. Gurtler, "Are Enterovirus Infection a Co-Factor in Sudden Hearing Loss,” Journal of Medical Virology, Vol. 72, No. 4, 2004, pp. 625-629. http://dx.doi.org/10.1002/jmv.20014

[11] M. Peltomaa, I. Pyykkö, I. Seppälä and M. Viljanen, "Lyme Borreliosis-An Unusual Cause of Vertigo," $\mathrm{Au}$ ris Nasus Larynx, Vol. 25, No. 3, 1998, pp. 233-242. http://dx.doi.org/10.1016/S0385-8146(97)10039-6

[12] Z. Selmani, H. Ishizaki and I. Pyykkö, "Can Low Frequency Sound Stimulation during Posturography help diagnosing possible Perilymphatic Fistula in Patients with Sensorineural Hearing Loss and/or Vertigo?” European Archives of Oto-Rhino-Laryngology, Vol. 261, No. 3, 2004, pp. 129-132.

[13] H. Aalto, I. Pyykkö and J. Starck, "Computerized Posturography, a Development of the Measuring System,” 
Acta Otolaryngol (Stockh), Vol. 105, No. s449, 1988, pp. 71-75. http://dx.doi.org/10.3109/00016488809106382

[14] H. Enbom, M. Magnusson, I. Pyykkö and L. Schalen, "Presentation of a Posturographic Test with Loading of the Proprioceptive System,” Acta Otolaryngol (Stockh), Vol. 105, No. s455, 1988, pp. 58-61.

http://dx.doi.org/10.3109/00016488809125058

[15] H. Richardson, J. P. Birchall, J. Hill and T. McMaster, "Should We Routinely Screen for Lyme Disease in Patients with Asymmetrical Hearing Loss?” British Journal of Audiology, Vol. 28, No. 2, 1994, pp. 59-61. http://dx.doi.org/10.3109/03005369409077915

[16] P. Wahlberg, H. Granlund, D. Nyman, J. Panelius and I. Seppälä, "Late Lyme Borreliosis: Epidemiology, Diagnosis and Clinical Features," Annals of Medicine, Vol. 25, No. 4, 1993, pp. 349-352.

[17] H. Ishizaki, I. Pyykkö, H. Aalto and J. Starck, "Tullio Phenomenon and Postural Stability. Experimental Study in Normal Subjects and Patients with Vertigo," Annals of Otology, Rhinology \& Laryngology, Vol. 100, No. 12, 1991, pp. 976-983. 